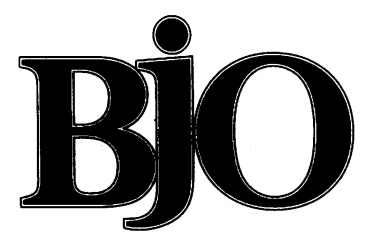

British Journal of Ophthalmology

\title{
Editorials
}

\section{Irradiating iris tumours}

Melanocytic tumours of the iris stroma are rare, enigmatic lesions with controversy surrounding both their diagnosis and subsequent management. While it is accepted that both naevi and true melanomas may arise within the iris stroma, it may be extremely difficult to distinguish between them clinically, or indeed histologically. It has been proposed that size, local replacement of the iris, prominent vascularity, ectropion iridis, secondary cataract, secondary glaucoma, and progressive growth are all features that may indicate malignancy. ${ }^{1}$ Although these factors are no doubt of value when reaching a diagnosis, especially when viewed collectively, they are not pathognomonic. For example, progressive enlargement, perhaps the most compelling pointer to malignancy, may be observed in benign lesions. Territto et $a l^{2}$ reviewed the outcome of 175 patients with melanocytic lesions which had been managed by observation for between 1 and 12 years. Five lesions were removed surgically because of documented growth. Of these, two were found to be benign naevi when examined histologically.

The ancillary investigations which have proved useful in establishing the nature of posterior uveal tumours, appear to be of limited value when applied to iris lesions. Traditional ultrasonography, because of the poor resolution of the anterior segment, is of no value in the diagnosis of iris melanocytic tumours. Similarly, the radioactive phosphorus uptake test has no role in the diagnosis of anterior uveal tumours. ${ }^{3}$ While fluorescein angiography may demonstrate the degree of vascularity within the tumour, ${ }^{4}$ its diagnostic impact is blunted by the fact that other neoplastic or inflammatory lesions may possess prominent leaking vessels. ${ }^{5}$ Recently, fine needle aspiration biopsy has been advocated as a useful method of establishing the diagnosis of iris lesions, ${ }^{6}$ despite the obvious limitations of inadequate sampling and discrepancies in the interpretation of the cytological material.

Iris melanomas appear to differ from ciliary body and choroidal melanomas in a number of respects: affected patients are generally 10-12 years younger than patients with melanomas of the posterior uvea, ${ }^{78}$ the lesions are usually smaller, and their metastatic potential is low. ${ }^{9}$ Geisse and Robertson ${ }^{9}$ reviewed the literature and found that out of 1043 reported iris melanomas, only 31 (3\%) had metastasised. The benign nature of these lesions has led some authorities to reappraise their histological classification. Jakobiec and Silbert ${ }^{8}$ in a retrospective clinicopathological study reclassified 189 iris or iris and ciliary body tumours into a new nine part histopathological classification. Only $13 \%$ of the original tumours were assigned to one of three malignant categories, the remaining $87 \%$ were considered to be benign lesions. A reclassification of all spindle cell A tumours from melanomas to spindle cell naevi was a quintessential element of this study. This arbitrary reappraisal has been challenged by some ${ }^{10}$ who point to the fact that documented deaths from metastases have resulted from pure spindle cell A tumours of the iris.

Even if one accepts the contentious histological nature of iris tumours, their overall prognosis, especially when compared with other uveal melanomas, is generally excellent. The iris melanocyte is histologically indistinguishable from other uveal melanocytes and shares the same embryonic origin. ${ }^{11}$ Why then should iris melanomas behave so differently? One obvious difference between iris lesions and other uveal melanomas is their relatively small size. Davidorf ${ }^{12}$ in a review of 53 iris tumours found that the average iris melanoma had a volume of only $55 \mathrm{~mm}^{3}$, this is considerably less then half the volume of 'small' choroidal tumours. He argued that when uveal tumours of equivalent size are considered, their prognosis is similar, irrespective of location.

Recognition of the benign nature of most iris melanocytic tumours has led to the adoption of a more conservative approach to their management. In the majority of cases immediate surgical intervention is not warranted and the patient may be managed by periodic observation. ${ }^{5}$ If growth is observed then, in the case of most circumscribed lesions, sector iridectomy or iridocyclectomy may be performed. Large diffuse tumours, those with extensive seeding of the iridocorneal angle, or with intractable secondary glaucoma are, in general, not amenable to surgical resection. In these cases enucleation of the affected eye has been the only feasible alternative. In this issue Shields et al (p 306) have proposed a novel approach to the management of large diffuse, surgically non-resectable tumours. Prompted by the success of radioactive plaque brachytherapy in the treatment of posterior uveal melanomas, they have used this technique to treat a series of large diffuse iris melanomas together with a smaller cohort of iris metastases. Traditionally, radiotherapy has not been advocated for the treatment of iris tumours because of the fear of damage to the cornea, lens, and other radiosensitive tissues. They have treated a total of 18 iris neoplasms ( 14 melanomas, four metastases) using this method and in all but one, tumour regression was observed. A number of radiation 
induced complications have occurred including cataract, corneal problems, hyphaema, and focal iris vasculopathy. The authors conclude that the study provides an adequate assessment of the early complications following plaque radiotherapy. They point to the fact that radiation complications usually occur in the first 12 to 24 months after plaque radiotherapy, and that the mean follow up for iris

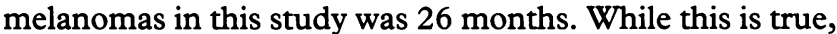
it is apparent from the results that complications, particularly cataract, tended to occur in those patients with the longest follow up and it is possible that further adverse effects may occur in time. Despite these complications, useful vision was preserved in a significant number of cases. Plaque radiotherapy appears to provide the clinician with a viable alternative to enucleation in the management of large diffuse iris melanomas.

Department of Ophthalmology and Orthoptics,

IAN RENNIE

University of Sheffield,

Royal Hallamshire Hospital,

Glossop Road, Sheffield S10 2JF
1 Shields JA, Sanborn GE, Augsburger JJ. The differential diagnosis of malignant melanoma of the iris. A clinical study of 200 patients. Ophthalmology 1983; 90: 716-20.

2 Territo C, Shields CL, Shields JA, Augsburger JJ, Schroeder RP Natural course of melanocytic tumors of the iris. Ophthalmology 1988; 95: 1251-5.

3 Shields JA. Accuracy and limitation of the P-32 test in the diagnosis of ocular tumours: an analysis of 500 cases. Ophthalmology 1978; 85: 950-66.

4 Jakobiec FA, Depot MJ, Henkind P, Spencer WH. Fluorescein angiographic patterns of iris melanocytic tumors. Arch Ophthalmol 1982; 100: $1288-99$.

5 Shields JA, Shields CL. Intraocular tumours. Philadelphia: WB Saunders, 1992: 61-83.

6 Grossniklaus HE. Fine-needle aspiration biopsy of the iris. Arch Ophthalmo 1992; 110: 969-76.

7 Jensen OA. Malignant melanoma of the uvea in Denmark, 1943-1952. Acto Ophthalmol 1963; 75 (suppl): 173.

8 Jakobiec FA, Silbert G. Are most iris 'melanomas' really nevi? A clinicopathologic study of 189 lesions. Arch Ophthalmol 1981; 99: 2117-32.

9 Geisse LJ, Robertson DM. Iris melanomas. Am $\mathcal{f}$ Ophthalmol 1985; 99; 638-48.

10 Kersten RC, Tse DT, Anderson R. Iris melanoma. Nevus or malignancy? Surv Ophthalmol 1985; 29: 423-33.

1 'Rahlilly $\mathrm{R}$. The prenatal development of the human eye. Exp Eye Res 1975; 21: 93-101.

12 Davidorf FH. The melanoma controversy. A comparison of choroidal, cutaneous, and iris melanomas. Surv Ophthalmol 1981; 25: 373-7

\section{Pterygium - an ophthalmic enigma}

Pterygia, the wing-shaped fleshy growths on the corneal limbus have been known to physicians for thousands of years. The term pterygium was introduced to the English language in 1875 by Walton. ${ }^{1}$ Despite being recognised for many years and being very common in some parts of the world, very little is known about the pathogenesis of the condition. This ignorance is reflected in the poor results of intervention and the wide range of treatments advocated.

It has been accepted for some time that environmental factors are responsible for the development of pterygium. ${ }^{2-4}$ More recently, it has become clear that ultraviolet light exposure is the most important environmental influence 56 and that high exposure in the second or third decade of life is particularly relevant to causation. ${ }^{7}$ Most pterygia develop on the nasal limbus ${ }^{89}$ and it has been proposed that this is attributable to reflected sunlight being preferentially focused at this point. ${ }^{10}$

The way in which ultraviolet light interacts with the limbus and cornea to produce a pterygium is unknown. The histopathology is non-specific. Hyaline degeneration and a low grade inflammatory reaction is what is regularly seen. ${ }^{11-14}$ This does little to suggest an underlying cause.

Genetic factors are also important. ${ }^{15}$ In particular environments some racial groups are affected more than others ${ }^{8}$ and there is a tendency for pterygia to occur in families. ${ }^{16-18}$

The presence of an unsightly uncomfortable lump on the surface of the eye is usually indication enough for surgical removal. Less commonly, a pterygium may interfere with vision, either by occluding the optical axis or by inducing astigmatism. ${ }^{19} 20$ Unfortunately, excision is often complicated by recurrence and recurrent pterygia are usually more troublesome than their antecedents. ${ }^{21-23}$

Various approaches as an alternative to, or adjunctive to, surgery have evolved. The use of ionising radiation, 24-26 heat, ${ }^{27}$ lasers, ${ }^{28-30}$ and antimetabolites ${ }^{31-35}$ have been advocated as adjuncts to excision. Furthermore, there has been a strong move to use a planned surgical repair with a flap of normal conjunctiva or limbus ${ }^{36-44}$ or a free graft. $^{36-44}$ Apparently, improved results with this form of repair have encouraged the implication of the ephemeral limbal stern cell in the aetiology and pathogenesis of pterygia. Just how these cells might be involved can only be debated in a theoretical sense because there is no way of identifying these important but elusive progenitor cells unequivocally, although they clearly exist.

The use of cellular and molecular biology techniques offers the prospect of a fresh perspective of many ancient diseases. Perhaps this will be so for pterygium. Karukonda et al have begun to apply these techniques. They have taken the first steps in looking at the fundamental cell biology of pterygia. The authors report in this issue (p 313) that pterygia do not contain large numbers of proliferating cells. Their work addresses a problem of clinical relevance. If cell proliferation is an important part of the pathology of pterygia cytostatic therapy is likely to be specifically effective. The lack of specificity of cytostatic measures such as irradiation and mitomycin $\mathrm{C}$ accounts for the previous complications which have been attributed to these treatments. ${ }^{25} 4546$ If cellular proliferation is not a feature of the pathology an alternative approach is required. Perhaps there is a regional disturbance of growth and differentiation which is best managed by replenishing the area with appropriately differentiating groups of cells and this accounts for the successes attributable to various forms of autogenous repair which have been reported recently. ${ }^{36-44}$

Department of Ophthalmology,

D COSTER

Flinders Medical Centre,

Bedford Park,

South Australia 5042

1 Walton HH. A practical treatise on diseases of the eye. 3rd ed. London: J and A Churchill, 1875.

2 Cameron ME. Pterygium throughout the world. Springfield, IL: Charles C Thomas, 1965.

3 Kamel S. The pterygium: its aetiology and treatment. Am $\mathcal{f}$ Ophthalmol 1954; 38: 682-8.

4 Darrel RW, Bachrach CA. Pterygium among veterans: an epidemiological study showing a correlation between frequency of pterygium and degree of exposure of ultraviolet in sunlight. Arch Ophthalmol 1963; 70: 158-69. exposure of ultraviolet in sunlight. Arch Ophthalmol 1963; 70: 158-69.
Moran DJ, Hollows FC. Pterygium and ultraviolet radiation: a positive correlation. Brf Ophthalmol 1984; 68: 343-6.

6 Taylor HR. Ultraviolet radiation and pterygium [Letter]. $\mathcal{F} A M A 1982 ; 247$ : 1698.

7 MacKenzie FD, Hirst LW, Battistutta D, Green A. Risk analysis in the development of pterygia. Ophthalmology 1992; 99: 1056-61.

8 Detels R, Dhir SP. Pterygium: a geographical study. Arch Ophthalmol 1967; 78: 485-91. 